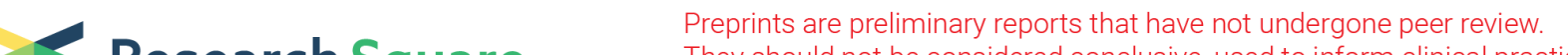 Research Square They should not be considered conclusive, used to inform clinical practice, or referenced by the media as validated information.
}

\section{Development of the "POP" scoring system for predicting obstetric and gynecological diseases in the emergency department: A retrospective cohort study}

ASAMI OKADA ( $\nabla$ asami.tsuji0515@gmail.com )

Kyoto Daini Red Cross Hospital https://orcid.org/0000-0001-8112-1397

Yohei Okada

Kyoto University

Hiroyuki Fujita

Kyoto Daini Red Cross Hospital

Ryoji liduka

Kyoto Daini Red Cross Hospital

Research article

Keywords: abdominal pain, emergency medicine, prediction model, gynecologic emergencies, screening

Posted Date: January 2nd, 2020

DOl: https://doi.org/10.21203/rs.2.19944/v1

License: (c) (i) This work is licensed under a Creative Commons Attribution 4.0 International License.

Read Full License

Version of Record: A version of this preprint was published at BMC Emergency Medicine on May 6th, 2020. See the published version at https://doi.org/10.1186/s12873-020-00332-z. 


\section{Abstract}

Background: Obstetric and gynecological (OBGY) diseases are among the most important differential diagnoses for young women with acute abdominal pain. However, there are few established clinical prediction rules for screening OBGY diseases in the emergency department (ED). This study aimed to develop a prediction model for diagnosing OBGY diseases in the ED.

Methods: This single-center retrospective cohort study included female patients with acute abdominal pain who presented to our emergency department. We developed a logistic regression model for predicting $O B G Y$ diseases and assessed its diagnostic ability. This study included young female patients aged between 16 and 49 years old, who had abdominal pain and were examined at the ED from April 2017 to March 2018. Trauma patients and patients referred from another hospital or from the OBGY department of our hospital were excluded.

Results: Of 27,991 patients, 740 were included. Sixty-five patients were diagnosed with OBGY diseases (8.8\%). The "POP" scoring system [past history of OBGY diseases +1 , no other symptoms +1 , and peritoneal irritation signs +1] was developed. Cut-off values set between 0 and 1 points, sensitivity at $97 \%$, specificity at $39 \%$, and negative likelihood ratio (LR-) of 0.08 were considered for rule-out, while cutoff values set between 2 and 3 points, sensitivity at $23 \%$, specificity at $99 \%$, and positive likelihood ratio $(\mathrm{LR}+)$ of 17.29 were suitable for rule-in.

Conclusions: Our "POP" scoring system can be useful for screening of OBGY diseases in the ED.

\section{Background}

Acute abdominal pain is one of the most common severe presentations in the emergency department (ED), and its differential diagnosis includes a very broad range of possible etiologies. Thus, a systematic diagnostic procedure is necessary to making an appropriate diagnosis. For young women with abdominal pain, obstetric and gynecological (OBGY) diseases such as ectopic pregnancy are some of the most important differential diagnoses. This is because a delayed diagnosis can be life-threatening, may affect their reproductive function, and decline the quality of life. $(1,2)$

Generally, a diagnosis for OBGY disease requires vaginal examinations or pelvic and transvaginal ultrasonographies by trained specialists such as OBGY physicians. $(3,4)$ However, the availability is limited in ED in Japan. Therefore, an easy screening tool to estimate the possibility of OBGY disease is necessary for appropriate consultation with an OBGY physician. Nevertheless, there are few established clinical prediction rules for screening emergency OBGY disease in the ED.

This study therefore aimed to develop and validate a prediction model for the diagnosis of OBGY diseases in the ED.

\section{Methods}


This study was a retrospective cohort study to develop the prediction model, which complied with the TRIPOD statement (Transparent Reporting of a Multivariable Prediction Model for Individual Prognosis or Diagnosis) regarding the reporting of the study's methods and results.(5) This study was approved by the Clinical Research Ethics Committee of Kyoto Daini Red Cross Hospital (Approval ID No. 2018-08). The ethics committee waived the requirement for informed consent because of the anonymous nature of the data.

\section{Data source and settings}

We obtained the clinical data by electrical chart review from the Japanese Red Cross Society Kyoto Daini Hospital Tertiary Critical Care Center in Kyoto City, Japan. Kyoto City is an urban area whose population is approximately 1.5 million, and the total number of ambulance requests per year is approximately 80,000 cases in the entire city.(6) There are four tertiary critical care centers in Kyoto City, and this hospital is one of those located in the center of Kyoto City. This hospital has 672 beds, and it provides primary to tertiary emergency care for any type of emergency cases such as severe trauma, cardiac arrest, and stroke. There are 7,679 ambulances, and walk-in visits in 2017 totaled to 20,312 cases. Hence, consultations with an OBGY physician on duty and an emergency surgeon are always available if necessary.

\section{Study population}

This study included young female patients aged between 16 and 49 years old, who had abdominal pain and were examined at the ED from April 2017 to March 2018. Trauma patients and patients referred from another hospital or from the OBGY department of our hospital were excluded.

\section{Data collection and patient outcomes}

We collected the following clinical information upon ED admission through the electrical chart review: age, time of hospital arrival, mode of ED visit (walk-in or ambulance), medical past history of OBGY disease, the symptoms [fever, digestive symptoms (e.g. vomiting, diarrhea)], atypical genital bleeding, and sign of peritoneal irritation upon physical examination. We defined medical history of OBGY disease as previous surgery due to gynecologic diseases such as ovarian, uterine, pelvic inflammatory, or sexually transmitted diseases. We also defined peritoneal irritation sign as muscular defense, guarding, rigidity, rebound tenderness, percussion tenderness, or heel drop test positive, based on the Japanese practice guideline for primary care of acute abdomen 2015.(7) We also collected final diagnosis at hospital discharge based on the International Statistical Classification of Diseases and Related Health Problems (ICD-10).

The primary outcome of interest was defined as final diagnosis of OBGY disease except for menstrual pain, which was determined by an OBGY or ED physician.

\section{Prognostic variable selection, handling missing data and sample size estimation}


Based on previous studies, $(1,8)$ our experience, and expert opinion, we selected three variables (past history of OBGY disease, no other symptom, and peritoneal irritation sign) as potential predictors of diagnostic of OBGY disease.

If missing values exist, missing data were categorized as "unknown" because unmeasured values might be informative in clinical settings. This study was a retrospective analysis, so sample size estimation was not required.

\section{Statistical analysis}

We described the patients' characteristics. Further, we conducted logistic regression analysis to calculate each variable's coefficient $ß$ and odds ratios (ORs) with 95\% confidence intervals (Cls). The model's performance was evaluated based on the C-statistics, the calibration intercept and slope, and the Brier score. Interval validation was estimated by a bootstrapping procedure using 1000 samples with replacement from the original sample. Finally, we set the clinically useful simplified screening system using a simple integer score based on each variable's coefficient $B$. The diagnostic abilities [sensitivity, specificity, positive likelihood ratio (LR+), and negative likelihood ratio (LR-)] of each score were calculated. The calibration performance of risk stratification was graphically evaluated in terms of the relationship between the predicted probability and observed proportion of the OBGY disease diagnosis. All statistical results were considered significant at two-sided $P$ values of $<0.05$. Statistical analyses were performed using JMP Pro®囚 14 software (SAS Institute Inc., Cary, NC) and R software (version 1.1.456; R Studio Inc.) with the "rms" package.(9)

\section{Results}

\section{Patient characteristics}

Among the 27,991 patients presented at the ED, 894 young female patients had acute abdominal pain. We excluded 112 patients who directly consulted an OBGY physician, 25 patients who were referred from another hospital, 12 patients with trauma, and 5 patients who were referred from the OBGY department in our hospital. Finally, 740 patients were included for the analysis (Fig. 1). Of these patients, except for menstrual pain $(\mathrm{N}=51), 65$ patients were diagnosed with OBGY disease $(8.8 \%)$. The characteristics of the patients are shown in Table 1. Details of OBGY diseases and all diseases are shown in the Table 2 and supplementary file 1.

\section{Performance and internal validation of the model}

The variables' coefficient $ß$, crude ORs with $95 \% \mathrm{Cl}$, adjusted ORs with $95 \% \mathrm{Cl}$, and formula for predicted diagnostic of OBGY disease are shown in the Table 3. As an internal validation, we also described the bias-corrected model performance using bootstrap procedure in the supplementary file 2 . It indicated that the risk of bias by overfitting was estimated as low. 
Based on the $\beta$ coefficient of each predictor, we created a prediction scoring system POP score [Past history of OBGY disease: +1 point, no $\underline{0}$ ther symptom: +1 point, and Peritoneal irritation sign: +1 point]. The c-statistics of this score was 0.794 . Particularly, rule-out was suitable if the cut-off values were set between 0 and 1 point, sensitivity was at $97 \%$, specificity was at $39 \%$, and LR- was 0.08 . Further, rule-in was suitable if the cut-off values were set between 2 and 3 points, sensitivity was at $23 \%$, specificity was at $99 \%$, and LR+ was 17.29 (Table 4). Additionally, graphical evaluation of the scoring system revealed good calibration between prediction and observation (Fig. 2).

\section{Discussion}

\section{Key observations}

The present study showed that the "POP" scoring system (Past history of OBGY, no Other symptoms, and Peritoneal irradiation sign) had good screening ability for OBGY disease, with good discrimination and calibration with internal validation in the ED setting.

\section{Previous literature and the present study's strengths}

Compared to previous studies, our study has some strengths for generalizability. A previous prospective multicenter study in five OBGY departments in Paris $(\mathrm{N}=516)$ developed and validated a clinical prediction rule for identifying life-threatening diseases (e.g. ectopic pregnancy, adnexal torsion or tuboovarian abscess which can lead to hemodynamic instability, organ failures, severe morbidity, and death) in gynecological emergency rooms for patients with acute pelvic pain. Vomiting, sudden onset of fever, and pain from palpation are significantly associated with life-threatening disease.(1) However, the setting of this previous study was specialized for gynecological emergency, which was substantially different from the primary care or general emergency departments as the setting in the previous study did not include various types of diseases (i.e., digestive or urological diseases). Thus, the generalizability may be limited (spectrum bias). Conversely, our study setting was general ED in an urban area. Therefore, our study had the strength in terms of generalizability as compared to other ED settings.

Other previous prospective studies in the United States developed and validated a prediction model for ectopic pregnancy in the ED.(8) In this previous study, patients were limited to early pregnant women who visited the ED, and predictors included cervical motion tenderness and fetal heart rate. For nongynecologist physicians, the opportunity to perform vaginal examinations or transvaginal ultrasonography is extremely limited in Japan. Thus, this model to predict ectopic pregnancy cannot be applied in general emergency departments. Accordingly, we believe that our prediction rule may be more suitable for diagnosing or excluding OBGY diseases in general ED.

\section{Interpretation}

We suggested the possible explanation of this prediction model. The present study evaluated clinically relevant variables that can be summarized as "POP" (past history of OBGY disease, other symptoms, and peritoneal irradiation sign). In terms of the past history of OBGY diseases, it is reported that ovarian 
tumor rupture and adnexal torsion are likely to occur and recur in patients with a history of ovarian tumor. $(2,10,11)$ Accordingly, past history of OBGY diseases is important clinical information for prediction. Moreover, previous studies reported that vomiting is associated tubal rupture and adnexal torsion. $(12,13)$ However, the study population was only composed of patients who were diagnosed with OBGY diseases. Conversely, most patients in our study (489/740: 66\%) were diagnosed with digestive diseases; half of them (250/489: $51 \%$ ) complained of vomiting, while only $12 \%$ (8/65) with OBGY diseases had vomiting. Hence, it may be reasonable that no vomiting was more associated with OBGY diseases than other cases especially related to digestive disease in general ED. Moreover, in terms of fever, there was no association between fever and OBGY diseases.(12) On peritoneal irritation signs, most patients with ectopic pregnancy had abdominal peritoneal signs.(8) Thus, we assumed that ovarian bleeding and ectopic pregnancy cause bleeding in the pelvic cavity, and PID causes localized inflammation in the pelvis. Similarly, we found that most patients hospitalized for OBGY disease or those who underwent emergency surgery for OBGY disease also had peritoneal irritation sign. Atypical genital bleeding can be expected to be associated with OBGY diseases. However, in this study, there were only 2 cases out of 740 cases with an atypical genital bleeding. Therefore, the association between atypical genital bleeding and OBGY diseases was unknown in our study.

Hence, it is reasonable that we selected these contents to be the clinical predictors.

\section{Clinical implications}

The clinical implications of this study are that OBGY diseases can diagnosed or excluded by this simple scoring system. When score cut-off was set at $0 / 1$ point, the negative likelihood ratio was 0.08 in our findings, which is useful to rule out OBGY diseases. If the prior probability is $8.8 \%$ was the same as in our setting, the posterior probability decreased to $1.3 \%$ when the score is 0 . As an expected advantage of easy screening to exclude OBGY disease diagnosis, we presumed that there would be decrease in unnecessary consultation and number of transfers from the hospital without obstetricians and gynecologists, thereby improving the workload of these specialist physicians. When score cut-off was set at $2 / 3$ points, the positive likelihood ratio was 17.25 . The posterior probability increased to $55 \%$ in the abovementioned setting, when the score was 3 . It may be useful for rule-in, leading to appropriate consultation. We suggest consultation with gynecologists if the POP score is 3 points. Meanwhile, if the POP score was 1 or 2 points, we considered evaluating the results from other additional tests (e.g., blood test, transabdominal ultrasonography, and computed tomography). Accordingly, the POP score may be useful for rule-out or rule-in of OBGY disease in an ED setting, similar to the results of our study.

\section{Limitations}

Our study has several limitations. Firstly, this is a retrospective study based on chart review, wherein the validity of the diagnosis, measurement factors, and the missed diagnosis might have led to the information bias. Secondly, direct visitation to OBGY department may have led to the selection bias. Thirdly, we could not assess the external validation as our study was conducted in a single center, with a relatively small sample size. Despite using the bootstrap procedure, our results indicated a low risk of 
bias by overfitting. Thus, further research is necessary to evaluate the external validation and applicability in other areas and multi-centers.

\section{Conclusion}

The "POP" scoring system had good discrimination and calibration for the diagnosis of OBGY diseases in young female patients with abdominal pain, who presented in the ED. The findings can therefore be useful for screening OBGY diseases in the ED. However, further research to assess the external validity is necessary.

\section{Declarations}

Ethics approval and consent to participate: This study was approved by the Clinical Research Ethics Committee of Kyoto Daini Red Cross Hospital (Approval ID No. 2018-08). The ethics committee waived the requirement for informed consent because of the anonymous nature of the data. We provided a means to opt out in our hospital.

Consent for publication: Not applicable.

Availability of data and materials: Data sharing is not applicable to this article as ethics committee did not approve it.

Competing interests: The authors do not have any competing interests related to this manuscript.

Funding: This research did not receive any specific grant from funding agencies in the public, commercial or not-for-profit sectors.

Authors' contributions: $\mathrm{AO}$ and $\mathrm{YO}$ contributed to the conception and design of this study, and $\mathrm{AO}$ wrote the manuscript. RI supervised the conception and design based on their specialty in ED management. HF directed the discussion part of the manuscript from the viewpoint of his specialty on obstetrics and gynecology. YO supervised the statistical method from the viewpoint of his specialty on epidemiology and statistics. All authors revised the draft critically, approved the contents of the manuscript, and agreed to be accountable for all aspects of the work.

Acknowledgments: Not applicable.

\section{Abbreviations}

OBGY - Obstetric and gynecological

ED - Emergency department

$\mathrm{OR}$ - odds ratio 


\section{References}

1. Huchon C, Dumont A, Chantry A, Falissard B, Fauconnier A. Triage using a self-assessment questionnaire to detect potentially life-threatening emergencies in gynecology. World J Emerg Surg. 2014;9:46.

2. Sasaki KJ, Miller CE. Adnexal torsion: review of the literature. J Minim Invasive Gynecol. 2014;21(2):196-202.

3. Moore CL, Copel JA. Point-of-care ultrasonography. N Engl J Med. 2011;364(8):749-57.

4. Bignardi T, Burnet S, Alhamdan D, Lu C, Pardey J, Benzie R, et al. Management of women referred to an acute gynecology unit: impact of an ultrasound-based model of care. Ultrasound Obstet Gynecol. 2010;35(3):344-8.

5. Collins GS, Reitsma JB, Altman DG, Moons KG. Transparent reporting of a multivariable prediction model for individual prognosis or diagnosis (TRIPOD): the TRIPOD statement. Bmj. 2015;350:g7594.

6. Department KCF. Kyoto City Fire Department囚Emergency statistics 2018/9/26 [Available from: https://www.city.kyoto.lg.jp/shobo/page/0000224446.html.

7. Yokoe M, Takada T, Mayumi T, Yoshida M, Isaji S, Wada K, et al. Japanese guidelines for the management of acute pancreatitis: Japanese Guidelines 2015. J Hepatobiliary Pancreat Sci. 2015;22(6):405-32.

8. Buckley RG, King KJ, Disney JD, Ambroz PK, Gorman JD, Klausen JH. Derivation of a clinical prediction model for the emergency department diagnosis of ectopic pregnancy. Acad Emerg Med. 1998;5(10):951-60.

9. Harrell JFE. Regression modeling strategies : with applications to linear models, logistic regression, and survival analysis. Frank E. Harrell, Jr., editor. New York: Springer; 2010.

10. Pansky M, Smorgick N, Herman A, Schneider D, Halperin R. Torsion of normal adnexa in postmenarchal women and risk of recurrence. Obstet Gynecol. 2007;109(2 Pt 1):355-9.

11. Pansky M, Feingold M, Maymon R, Ben Ami I, Halperin R, Smorgick N. Maternal adnexal torsion in pregnancy is associated with significant risk of recurrence. J Minim Invasive Gynecol. 2009;16(5):551-3.

12. Houry D, Abbott JT. Ovarian torsion: a fifteen-year review. Ann Emerg Med. 2001;38(2):156-9.

13. Huchon C, Panel P, Kayem G, Bassot A, Nguyen T, Falissard B, et al. Is a standardized questionnaire useful for tubal rupture screening in patients with ectopic pregnancy? Acad Emerg Med. 2012;19(1):24-30.

\section{Tables}

Table 1. Characteristics of the study participants 


\begin{tabular}{|c|c|c|c|c|c|c|}
\hline \multirow[t]{2}{*}{ Parameters } & \multirow{2}{*}{$\begin{array}{c}\text { Total } \\
(\mathrm{N}=740)\end{array}$} & \multicolumn{5}{|c|}{ Final diagnosis } \\
\hline & & $\begin{array}{c}\text { OBGY } \\
\text { diseases } \\
(\mathrm{N}=65)\end{array}$ & $\begin{array}{l}\text { Menstrual } \\
\text { pain }(\mathrm{N}=51)\end{array}$ & $\begin{array}{l}\text { Digestive } \\
\text { diseases } \\
(\mathrm{N}=489)\end{array}$ & $\begin{array}{c}\text { Urological } \\
\text { diseases } \\
(\mathrm{N}=31)\end{array}$ & $\begin{array}{l}\text { Others } \\
(\mathrm{N}=104)\end{array}$ \\
\hline Age, median, (IQR) & $\begin{array}{c}30 \\
(23.0- \\
39.0)\end{array}$ & $\begin{array}{c}30(22.0- \\
40.5)\end{array}$ & $\begin{array}{c}24(20.0- \\
32.0)\end{array}$ & $30(23.0-39.0)$ & $33(24.0-41.0)$ & $\begin{array}{c}31(23.0- \\
39.75)\end{array}$ \\
\hline$<20$ years, $n,(\%)$ & $\begin{array}{c}76 \\
(10.3 \%)\end{array}$ & $3(4.6 \%)$ & $6(11.8 \%)$ & $56(11.5 \%)$ & $0(0.0 \%)$ & $\begin{array}{c}11 \\
(10.6 \%)\end{array}$ \\
\hline $20-29$ & $\begin{array}{c}278 \\
(37.6 \%)\end{array}$ & $25(38.5)$ & $28(54.9 \%)$ & $176(36.0 \%)$ & $12(38.7 \%)$ & $\begin{array}{c}37 \\
(35.6 \%)\end{array}$ \\
\hline $30-39$ & $\begin{array}{c}205 \\
(27.7 \%)\end{array}$ & $19(29.2 \%)$ & $9(17.6 \%)$ & $136(27.8 \%)$ & $11(35.5 \%)$ & $\begin{array}{c}30 \\
(28.8 \%)\end{array}$ \\
\hline$>40$ & $\begin{array}{c}181 \\
(24.5 \%)\end{array}$ & $18(27.7 \%)$ & $8(15.7 \%)$ & $121(24.7 \%)$ & $8(25.8 \%)$ & $\begin{array}{c}26 \\
(25.0 \%)\end{array}$ \\
\hline \multicolumn{7}{|l|}{ How to visit } \\
\hline Walk in, $n,(\%)$ & $\begin{array}{c}591 \\
(79.9 \%)\end{array}$ & $47(72.3 \%)$ & $16(31.4 \%)$ & $412(84.3 \%)$ & $26(83.9 \%)$ & $\begin{array}{c}14 \\
(13.5 \%)\end{array}$ \\
\hline Ambulance & $\begin{array}{c}149 \\
(20.1 \%)\end{array}$ & $18(27.7 \%)$ & $35(68.6 \%)$ & $77(15.7 \%)$ & $5(16.1 \%)$ & $\begin{array}{c}90 \\
(86.5 \%)\end{array}$ \\
\hline \multicolumn{7}{|c|}{ Past medical history of OBGY diseases } \\
\hline Yes, n, (\%) & $\begin{array}{c}153 \\
(20.7 \%)\end{array}$ & $32(49.2 \%)$ & $14(27.5 \%)$ & $84(17.2 \%)$ & $4(12.9 \%)$ & $\begin{array}{c}19 \\
(18.3 \%)\end{array}$ \\
\hline No & $\begin{array}{c}587 \\
(79.3 \%)\end{array}$ & $33(50.8 \%)$ & $37(72.5 \%)$ & $405(82.8 \%)$ & $27(87.1 \%)$ & $\begin{array}{c}85 \\
(81.7 \%)\end{array}$ \\
\hline \multicolumn{7}{|l|}{ Other symptoms } \\
\hline $\begin{array}{l}\text { Digestive } \\
\text { symptoms, n, (\%) }\end{array}$ & $\begin{array}{c}292 \\
(39.5 \%)\end{array}$ & $7(10.8 \%)$ & $5(9.8 \%)$ & $250(51.1 \%)$ & $8(25.8 \%)$ & $\begin{array}{c}22 \\
(21.2 \%)\end{array}$ \\
\hline Fever & $\begin{array}{c}32 \\
(4.3 \%)\end{array}$ & $2(3.1 \%)$ & $0(0.0 \%)$ & $26(5.3 \%)$ & $1(3.2 \%)$ & $3(2.9 \%)$ \\
\hline Nothing & $\begin{array}{c}386 \\
(52.2 \%)\end{array}$ & $56(86.2 \%)$ & $45(88.2 \%)$ & 203 (41.5\%) & $13(41.9 \%)$ & $\begin{array}{c}68 \\
(65.4 \%)\end{array}$ \\
\hline Other & $\begin{array}{c}30 \\
(4.1 \%)\end{array}$ & $0(0.0 \%)$ & $1(2.0 \%)$ & $10(2.0 \%)$ & $9(29.0 \%)$ & $\begin{array}{c}11 \\
(10.6 \%)\end{array}$ \\
\hline $\begin{array}{l}\text { Atypical genital } \\
\text { bleeding, n, (\%) }\end{array}$ & $2(0.3 \%)$ & $1(1.5 \%)$ & $0(0.0 \%)$ & $0(0.0 \%)$ & $0(0.0 \%)$ & $1(1.0 \%)$ \\
\hline $\begin{array}{l}\text { Peritoneal irritation } \\
\text { signs, } \mathrm{n},(\%)\end{array}$ & $\begin{array}{c}119 \\
(16.1 \%)\end{array}$ & $30(46.2 \%)$ & $2(3.9 \%)$ & 79 (16.2\%) & $2(6.5 \%)$ & $6(5.8 \%)$ \\
\hline Admission, $\mathrm{n},(\%)$ & $\begin{array}{c}81 \\
(10.9 \%)\end{array}$ & $22(33.8 \%)$ & $0(0.0 \%)$ & 56 (11.5\%) & $1(3.2 \%)$ & $2(1.9 \%)$ \\
\hline $\begin{array}{l}\text { Emergency } \\
\text { operation, n, (\%) }\end{array}$ & $\begin{array}{c}38 \\
(5.1 \%) \\
\end{array}$ & $14(21.5 \%)$ & $0(0.0 \%)$ & $24(4.9 \%)$ & $0(0.0 \%)$ & $0(0.0 \%)$ \\
\hline
\end{tabular}

IQR: interquartile range, OBGY: obstetric and gynecological.

\section{Table 2. Details of OBGY disease}




\begin{tabular}{lc}
\hline Details of OBGY diseases & $\mathbf{N = 6 5}$ \\
\hline Rupture of ovarian tumor & $12(18.5 \%)$ \\
\hline PID & $12(18.5 \%)$ \\
\hline Ovarian bleeding & $8(12.3 \%)$ \\
\hline Adnexal torsion & $7(10.8 \%)$ \\
\hline Uterine myoma & $6(9.2 \%)$ \\
\hline Ovulation pain & $5(7.7 \%)$ \\
\hline Ectopic pregnancy & $3(4.6 \%)$ \\
\hline Malignant tumor & $1(1.5 \%)$ \\
Abortion & $1(1.5 \%)$ \\
\hline Other & $10(15.4 \%)$ \\
\hline
\end{tabular}

OBGY: obstetric and gynecological, PID: pelvic inflammatory disease.

Table 3. $\beta$ coefficient, CORs, and AORs for each predictor

\begin{tabular}{lcccc}
\hline Predictors & $\begin{array}{c}\beta \\
\text { coefficient }\end{array}$ & CORs (95\% CI) & $\begin{array}{c}\beta \\
\text { coefficient }\end{array}$ & AORs (95\% CI) \\
\hline $\begin{array}{l}\text { Past history of OBGY } \\
\text { disease }\end{array}$ & 0.75 & $4.44(2.63-7.50)$ & 0.65 & $3.69(2.11-6.47)$ \\
No other symptoms & 0.88 & $\begin{array}{c}5.78(2.90- \\
11.54)\end{array}$ & 0.80 & $\begin{array}{c}4.95(2.43- \\
10.10)\end{array}$ \\
Peritoneal irritation signs & 0.87 & $5.64(3.30-9.65)$ & 0.80 & $4.96(2.80-8.79)$ \\
\hline
\end{tabular}

COR: crude odds ratio, AOR: adjusted odds ratio, CI: confidence intervals, OBGY: obstetric and gynecological.

Table 4. Diagnostic ability for each cut-off

\begin{tabular}{lcccccccc}
\hline Score cut-off & Sensitivity & Specificity & LR+ & LR- & TP & FP & TN & FN \\
\hline $\mathbf{3 / 2}$ & 0.23 & 0.99 & 17.29 & 0.78 & 15 & 9 & 666 & 50 \\
$\mathbf{2} / \mathbf{1}$ & 0.60 & 0.82 & 3.40 & 0.49 & 39 & 119 & 556 & 26 \\
\hline $1 / 0$ & 0.97 & 0.39 & 1.59 & 0.08 & 63 & 411 & 264 & 2
\end{tabular}

LR+: positive likelihood ratio, LR-: negative likelihood ratio, TP: true positive, FP: false positive, TN: true negative, FN: false negative, CI: confidence interval.

\section{Figures}




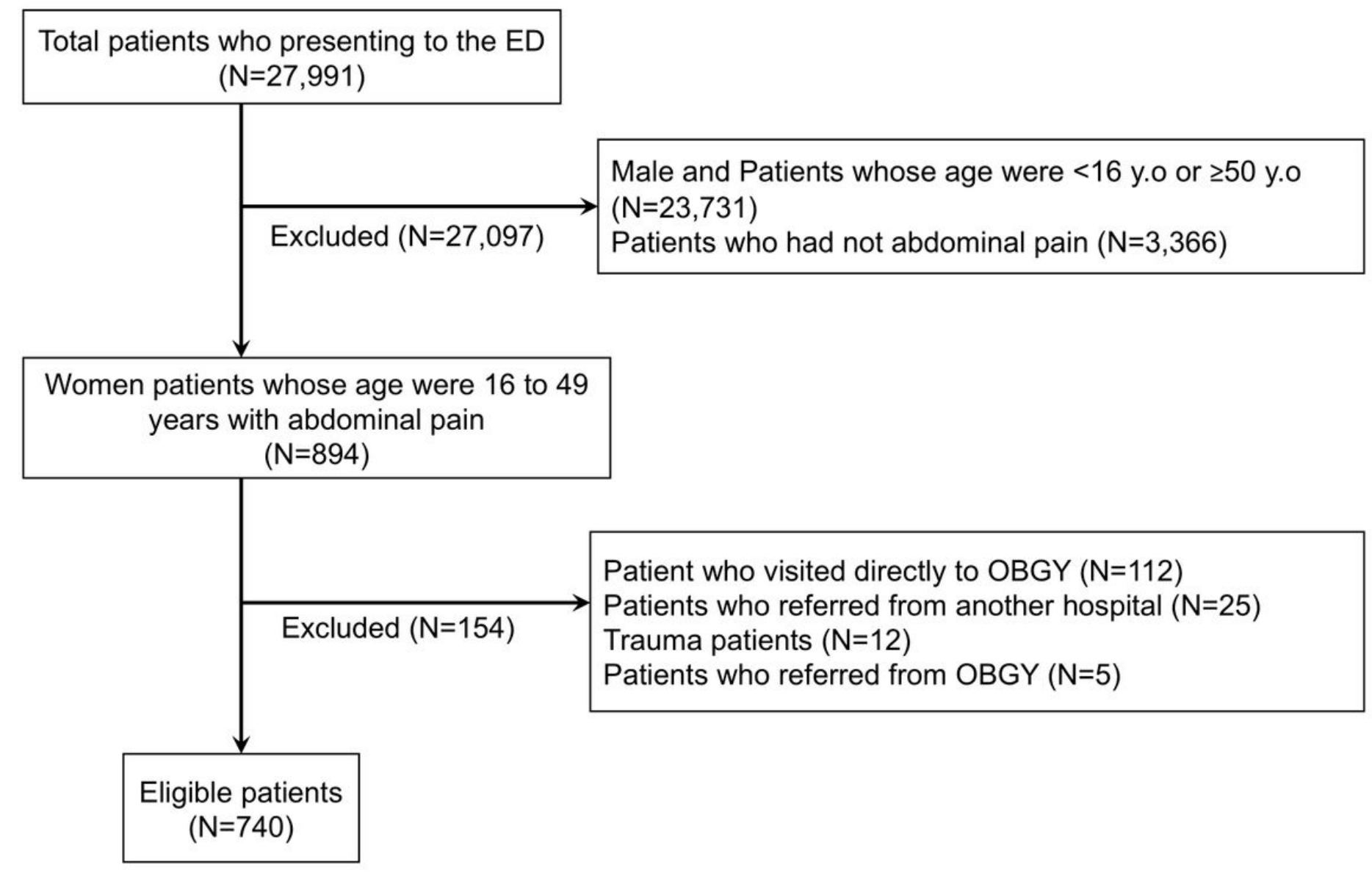

Figure 1

ED: emergency department, OBGY: obstetric and gynecological. 
100

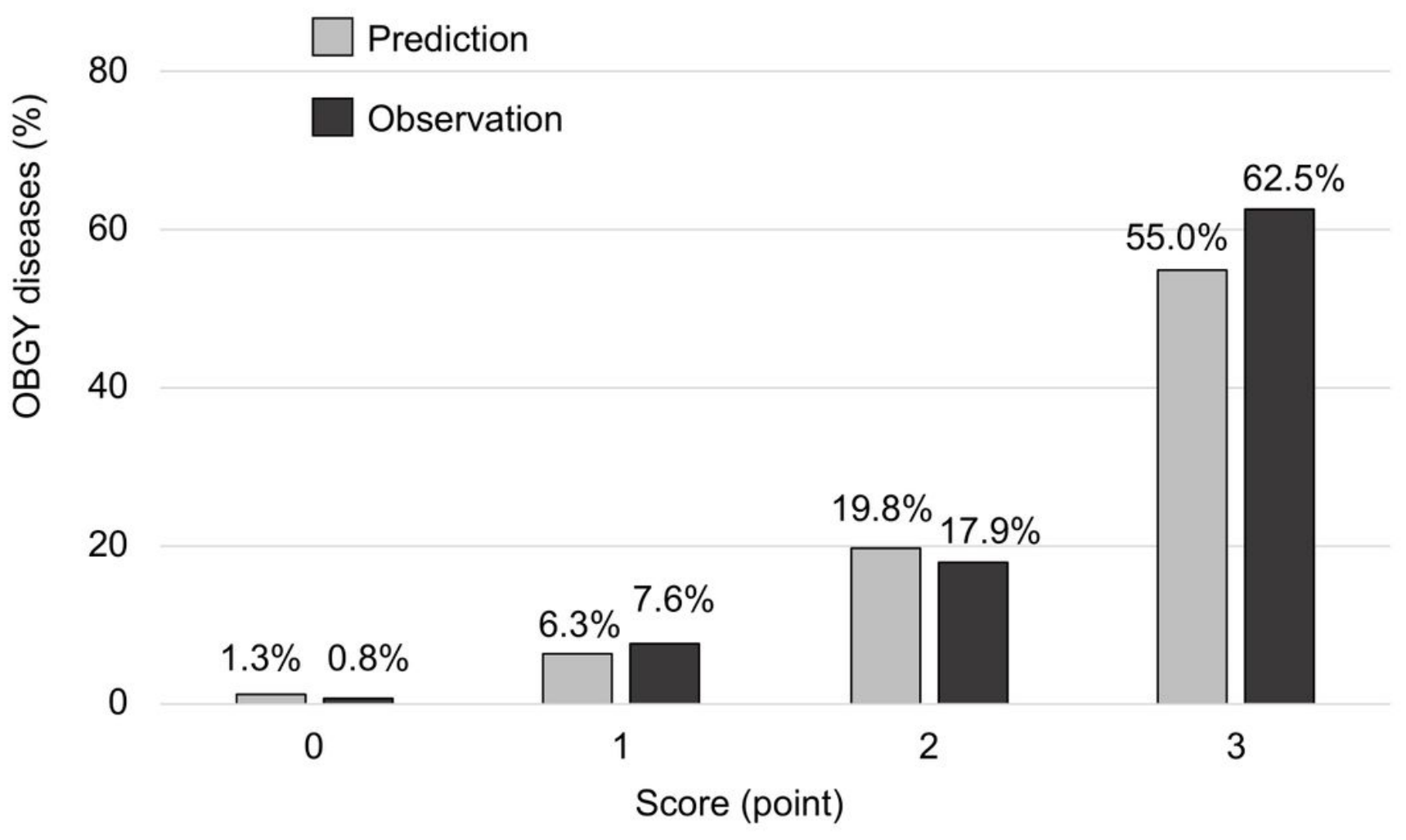

Figure 2

Predicted probability and observed proportion of OBGY diseases based on the POP scoring system. The mean predicted probability is shown by for the sums of the scores in each cohort. Observation reflected the observed proportion of diagnosed OBGY diseases. The predictions were well calibrated with the observations. The POP scoring system provided a simple and rapid prediction of OBGY diseases in ED. OBGY: obstetric and gynecological.

\section{Supplementary Files}

This is a list of supplementary files associated with this preprint. Click to download.

- SupplementaryFile.docx 